\title{
MiR-1254 inhibits proliferation, migration and invasion of human brain tumour cell lines
}

\author{
Gang Li $^{1}$, Dong-Gang Zhao', Lai Jiang ${ }^{1}$, Yu-Hua Guan ${ }^{1}$, Hua Tang ${ }^{1}$, Gang \\ Zheng $^{1}$, Xin Huang ${ }^{1}$, Yan Wang ${ }^{2 *}$ \\ ${ }^{1}$ Department of Neurosurgery, ${ }^{2}$ Department of Gynaecology, The First People's Hospital of Yichang, China Three Gorges \\ University, Yichang, Hubei 443000, China
}

*For correspondence: Email: WyllieCckech@yahoo.com

\begin{abstract}
Purpose: To investigate the expression of miR-1254 in 5 astrocytoma cell lines, and the mechanism involved.

Methods: Total RNA was isolated by RNeasy RNA isolation kit while cDNA was prepared by RevertAid $c D N A$ synthesis kit. The transcripts were analysed by real-time polymerase chain reaction (RT-PCR). Transfection of miR-1254 was carried out using FuGENE HD (Promega). Apoptosis was determined by $D A P I$, acridine orange (AO)/ethidium bromide (EB) and annexin V/PI double staining. Cell migration and invasion were investigated by wound healing and Martigel invasion assays, respectively. Protein expression was measured by western blotting.

Results: Expression of miR-1254 was significantly down-regulated in the astrocytoma cell lines when compared to normal astrocyte cells ( $p<0.05$ ). Ectopic expression of miR-1254 in astrocytoma SW 1088 cells inhibited cell proliferation via initiation of apoptosis and cell cycle arrest. Over-expression of miR1254 also led to significant decrease in cell migration and invasion of SW 1088 astrocytoma cells ( $p<$ 0.05).

Conclusion: The results show that the expression of miR-1254 is down-regulated in astrocytoma cell lines, but over-expression of miR-1254 inhibits proliferation of the cell lines via cell cycle arrest and apoptosis. Thus, miR-1254 has promising potential for use in the treatment of brain tumour.
\end{abstract}

Keywords: Brain tumour, Astrocytoma, miR-1254, Apoptosis, Cell migration

\begin{abstract}
This is an Open Access article that uses a funding model which does not charge readers or their institutions for access and distributed under the terms of the Creative Commons Attribution License (http://creativecommons.org/licenses/by/4.0) and the Budapest Open Access Initiative (http://www.budapestopenaccessinitiative.org/read), which permit unrestricted use, distribution, and reproduction in any medium, provided the original work is properly credited.

Tropical Journal of Pharmaceutical Research is indexed by Science Citation Index (SciSearch), Scopus, International Pharmaceutical Abstract, Chemical Abstracts, Embase, Index Copernicus, EBSCO, African Index Medicus, JournalSeek, Journal Citation Reports/Science Edition, Directory of Open Access Journals (DOAJ), African Journal Online, Bioline International, Open-J-Gate and Pharmacy Abstracts
\end{abstract}

\section{INTRODUCTION}

Micro RNAs (miRNAs) are small, non-coding RNA molecules that play vital roles in gene silencing. These miRNAs are about $18-25$ nucleotides in length and they control protein synthesis by targeting mRNAs through complementary binding [1]. The expression of miRNAs is often changed by diverse human disease conditions which include, but are not limited to, cancer [2]. Indeed, miRNAs are considered important in cancer. There are strong 
evidence for the involvement of miRNAs in proliferation, metastasis, angiogenesis, migration and invasion of cancer cells [2].

It has been reported that miRNAs can control these processes at the site of their origin or at sites remote from their sites of origin. The miRNAs influence the pathophysiology of diverse types of cancers [3]. The expression of miRNAs may either be up-regulated or down-regulated in cancerous tissues. However, it is has been reported that miRNAs show distinct types of expression profiles in different cancer types. The expression of miRNA may be up-regulated in one cancer type, but it may be down-regulated in other type [4]. Micro RNAs have been associated with tumorigenesis, in which they act as suppressors or oncogenes [5].

Several miRNAs have also been implicated in development of drug resistance in cancer cells. Among the miRNAs, miR-1254 has been shown to play crucial function in suppression of cell migration in several types of cancers such as colorectal cancer, adenocarcinoma carcinoma and lung cancer [6]. However, the expression profile of miR-1254 in brain tumour has not been investigated. In particular, its role in the development of the bone tumours has not been studied. Brain tumour which is often aggressive and rapidly-spreading, refers to an abnormal mass or growth in any region of the brain. It can be life-threatening, depending on the type, size, volume, location and origin [7]. The present study was therefore carried out to determine the expression of miR-1254 in five different astrocytoma cell lines and in normal human astrocytes.

\section{EXPERIMENTAL}

\section{Chemicals, reagents and cell cultures}

All reagents were obtained from Sigma-Aldrich Co. (St. Louis, MO, USA). Primary and secondary antibodies were obtained from Santa Cruz Biotechnology Inc. (Santa Cruz, CA, USA). Five human astrocytoma cell lines (CCF-STTG1, SW 1088, SW 178, U-87 M and U-118 M) were obtained from Type Culture Collection of Chinese Academy of Sciences, Shanghai, China. The cells were grown in RPMI-1640 medium containing $10 \%$ fetal bovine serum (FBS) and $100 \mathrm{U} / \mathrm{mL}$ (each) of penicillin and streptomycin, in a humidified atmosphere containing $5 \% \mathrm{CO}_{2}$.

\section{RNA isolation and synthesis of cDNA}

RNA was extracted from the five astrocytoma cell lines using TRIzol (Invitrogen) as per the manufacturer's protocol. Quality check of RNA samples was done by measuring absorbance using NanoDrop ${ }^{\circledR}$ ND-1000 spectrophotometer (NanoDrop Technologies), and also by separation on $1 \%$ agarose gel. DNA contamination was removed by DNase I (Fermentas) treatment. Thereafter, cDNA synthesis was done with the help of RevertAid cDNA synthesis kit (Fermentas).

\section{Quantification of relative expression of miR- 1254}

The expression profile of miR-1254 was studied using quantitative RT-PCR in the five astrocytoma cell lines (CCF-STTG1, SW 1088, SW 178, U-87 $\mathrm{M}$ and $\mathrm{U}-118 \mathrm{M}$ ) and normal human astrocytes. The reaction was performed in a total volume of $20 \mu$ l. The reaction mixture contained $10 \mu \mathrm{L}$ of $2 X$ SYBR Green Master Mix, $0.2 \mu \mathrm{M}$ primers and $100 \mathrm{ng}$ of cDNA. The cycling conditions were $95^{\circ} \mathrm{C}$ for $20 \mathrm{~s}$, followed by 40 cycles at $95{ }^{\circ} \mathrm{C}$ for $15 \mathrm{~s}$ and $58{ }^{\circ} \mathrm{C}$ for $1 \mathrm{~min}$. Actin amplification was used as control to normalize the data.

\section{Transfection}

For transfection of miR-1254, its upstream and downstream flanking sequences were amplified with the help of a genomic PCR. This was followed by cloning in pMSCv-pur vector. Thereafter the vector containing miR-1254 and the empty vector (used as negative control) were transfected into the SW 178 astrocytoma cells with help of FuGENE HD (Promega) as per the manufacturers' protocol.

\section{Cell viability assay}

Cell viability was assessed spectrophotometrically using MTT assay. Cultured SW 1088 cells transfected with pMSCv-pur vector containing miR-1254 or empty vector were seeded at a density of $1.2 \times 10^{4}$ to $1.5 \times 10^{4}$ in 96-well microtitre plates. This was followed by the addition of MTT solution to the wells. Absorbance at $570 \mathrm{~nm}$ was determined at 0,24 and $48 \mathrm{~h}$ in an ELISA plate reader.

\section{Apoptotic assay}

SW1088 astrocytoma cells were seeded at a density of $2 \times 10^{5}$ cells/well in 6 -well plates. The cells were then stained with a mixture of $A O$ and EB. The stained cells were examined under the fluorescent microscope. Another batch of cells was incubated with DAPI, and thereafter the cells were washed with PBS and fixed in $10 \%$ formaldehyde. The DAPI-stained cells were 
examined by fluorescent microscopy. The SW1088 brain tumour astrocytoma cells were also subjected to annexin V/PI and examined by flow cytometry.

\section{Cell cycle analysis}

In the estimation of the distribution of SW 1088 astrocytoma cells in different phases of cell cycle, the cells were fixed in $70 \%$ ethanol for about 1 hour and then PBS-washed. The cells were then suspended in a solution of $\mathrm{PI}$ (50 $\mu \mathrm{l} / \mathrm{mL})$ and RNase1 $(250 \mu \mathrm{g} / \mathrm{mL})$. This was followed by incubation for a period of $25 \mathrm{~min}$ at room temperature. Cell cycle analysis was carried out in fluorescence-activated cell sorting cater-plus cytometer using 10, 000 cells/group.

\section{Matrigel invasion assay}

Invasion was determined using Matrigel( $\mathrm{B}-\mathrm{coated}$ invasion chambers (BD Biosciences). The SW 1088 cells (transfected with pMSCv-pur vector containing miR-1254 or the empty vector) that reached the lower surface of the membrane were stained with crystal violet (CV). Images of CVstained cells were captured by a phase contrast microscope. The resultant crystal violet complex was dissolved in $10 \%$ acetic acid, and the extent of invasion was determined by measuring absorbance at $600 \mathrm{~nm}$.

\section{Western blot analysis}

Total protein from SW 1088 cells (transfected with pMSCv-pur vector containing miR-1254 or the empty vector) was isolated in RIPA lysis buffer. Equal volumes of the proteins from each sample were run on SDS PAGE. This was followed by transference to a polyvinylidene fluoride membrane, and blocking with $5 \%$ nonfat milk, and incubation at room temperature for 1 h. The membranes were then treated with specific primary antibody at $4{ }^{\circ} \mathrm{C}$ for $20 \mathrm{hrs}$. Thereafter, the membranes were washed in washing buffer, and incubated with secondary antibody for $1 \mathrm{~h}$. The protein bands were then visualised by an ECL Advanced Western blot detection kit.

\section{Statistical analysis}

All data are expressed as mean \pm SD. Statistically significant differences between groups were determined by one-way ANOVA and Tukey's post hoc test using GraphPad prism 7 software. Values of $p<0.05$ were considered as indicative of statistically significant differences.

\section{RESULTS}

\section{MiR-1254 was down-regulated in astrocytoma cell lines}

The expression of miR-1254 was significantly ( $p$ $<0.05)$ down-regulated in all the astrocytoma cell lines (Figure 1). The lowest expression was observed in CCF-STTG1, while the highest expression was observed in SW 178 cell line. Astrocytoma cell line SW 1088 showed moderate expression and was selected for further experimentation.

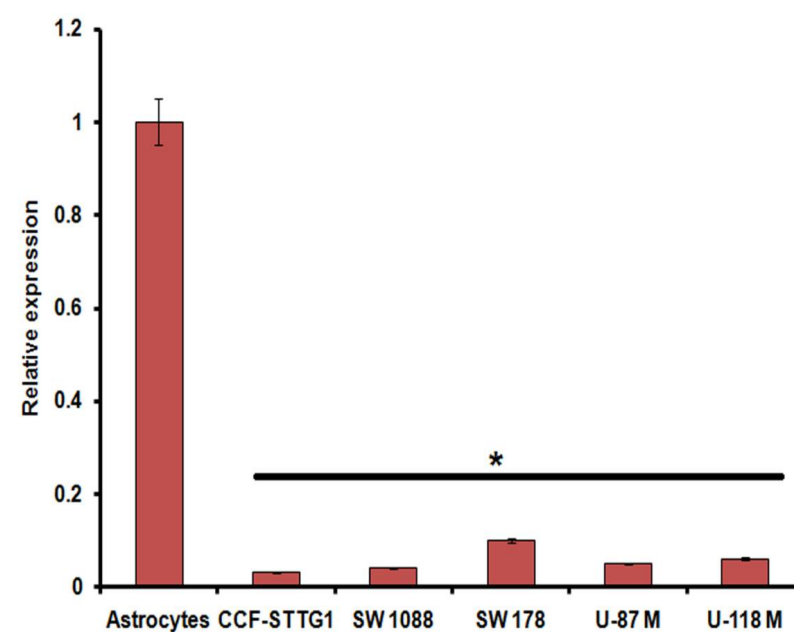

Figure 1: Expression of miR-1254 brain tumor astrocytoma cell lines and normal astrocytes as determined quantitative RT-PCR. The experiments were carried out thrice in triplicates and presented as mean $\pm \operatorname{SD}\left({ }^{*} p<0.05\right)$

\section{Ectopic expression of miR-1254 decreased cell viability through apoptosis}

To find out the role of miR-1254 in astrocytoma, the SW1088 cells were transfected with either empty vector or with vector containing miR-1254. The results showed that cells transfected with miR-1254 exhibited less cell viability when compared with the empty vector, control cells (Figure 2). These results indicate that ectopic expression of miR-1254 in SW1088 cells inhibited the viability of the cells. In order to investigate the underlying mechanism of the viability inhibition, DAPI, EB/OR and AnnexinV/PI staining were carried. The results obtained showed that the over-expression of miR-1254 in SW 1088 cells triggered apoptosis (Figure 3). Thus, miR-1254 reduced cell viability in miR1254 via induction of apoptosis.

\section{Ectopic expression of miR-1254 triggered G2/M cell cycle arrest}


The effect of miR-1254 on cell cycle distribution of SW 1088 astrocytoma cells was investigated. It was observed that cells that received vector containing miR-1254 showed increased number of cells in G2/M phase of the cell cycle, when compared with cells that received empty vector (Figure 4). These results suggest that miR-1254 exerted anti-proliferative effects, partly via induction of G2/M cell arrest of SW 1088 astrocytoma cells.

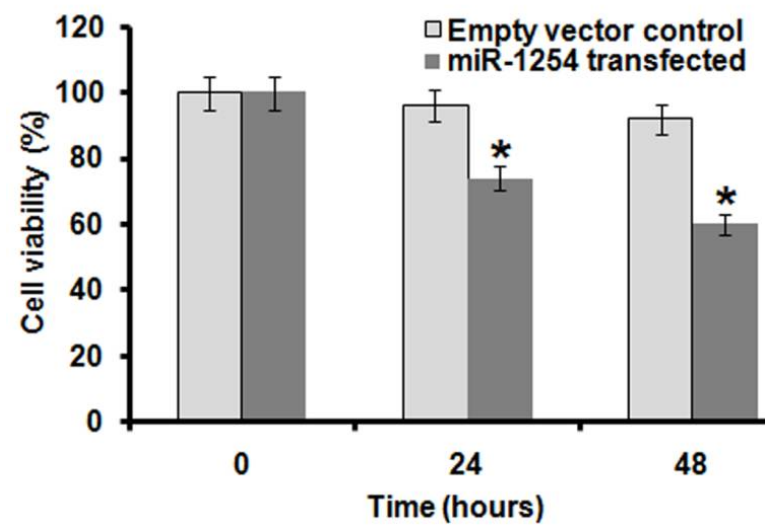

Figure 2: Cell viability of miR-1254 transfected and empty vector transfected SW 1088 cell line as determined by MTT assay. The experiments were carried out thrice in triplicates and presented as mean $\pm \mathrm{SD}\left({ }^{*} p<0.05\right)$

\section{Ectopic expression of miRNA-1254 inhibited cell migration and invasion}

Since, microRNAs have been reported to play important role in controlling the migration and invasion of cancer cells, the effect of miR-1254 on the cell migration and invasion of SW 1088 astrocytoma cells was investigated. The results of wound healing assay indicated that ectopic expression of miR-1254 significantly reduced migration of the SW 1088 cells after $20 \mathrm{~h}$. Within

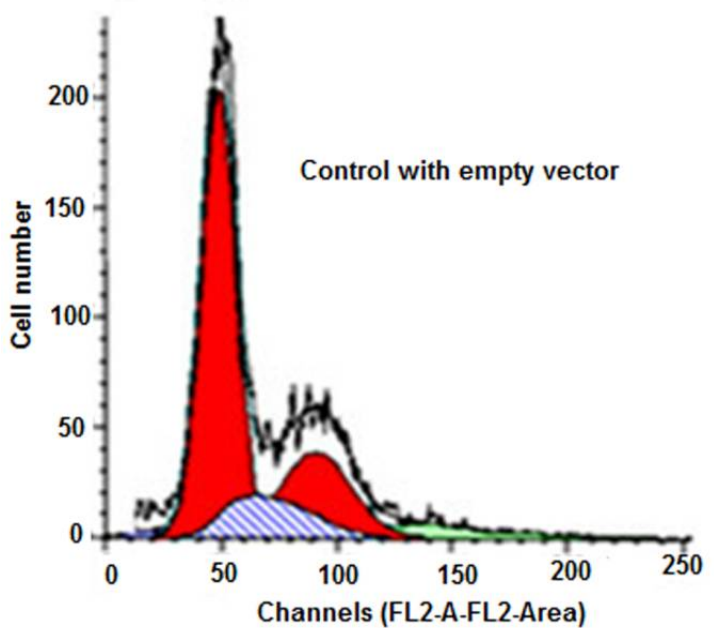

the same period, the relative migration in the control cells with empty vector was $31 \%$, when compared with $19 \%$ migration in the cells that over-expressed miR-1254 (Figure 5A \& 5B). Results of Martigel invasion assay showed that invasion in the astrocytoma SW 1088 that received vector was only $60 \%$, relative to control SW 1088 cells that received empty vector (Figure $5 \mathrm{C}$ and $5 \mathrm{D}$ ). These results clearly indicate that miR-1254 over-expression in SW 1088 cells inhibited cell migration and invasion.

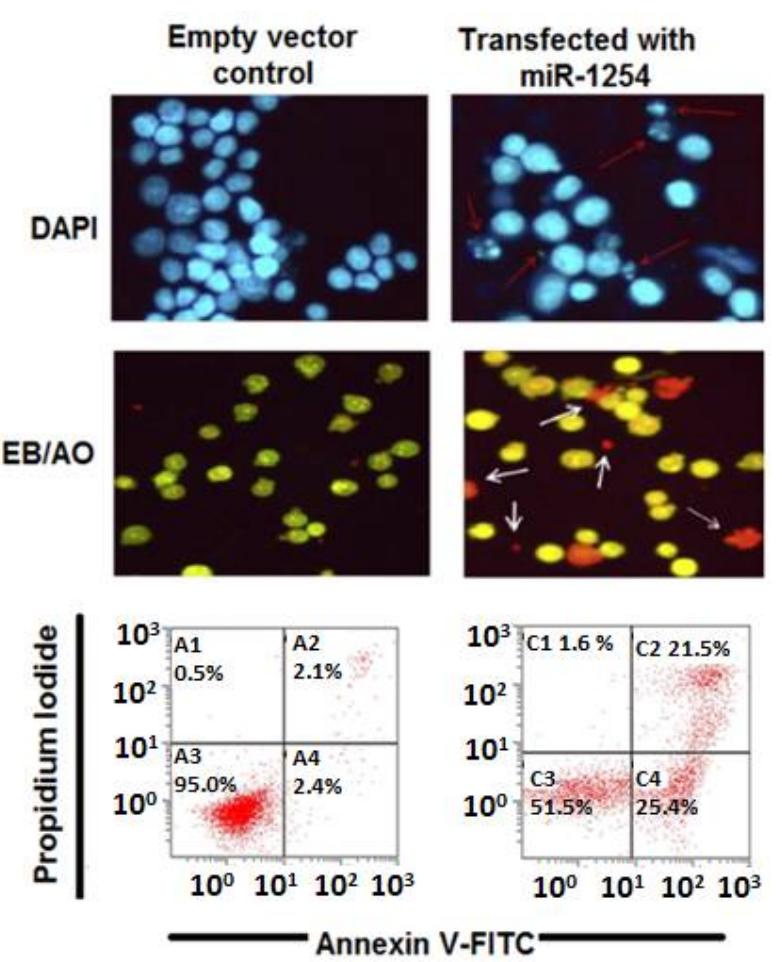

Figure 3: MiR-1254 overexpression triggers apoptosis as determined by DAPI, EB/AO and annexin V/PI double staining $(n=3)$

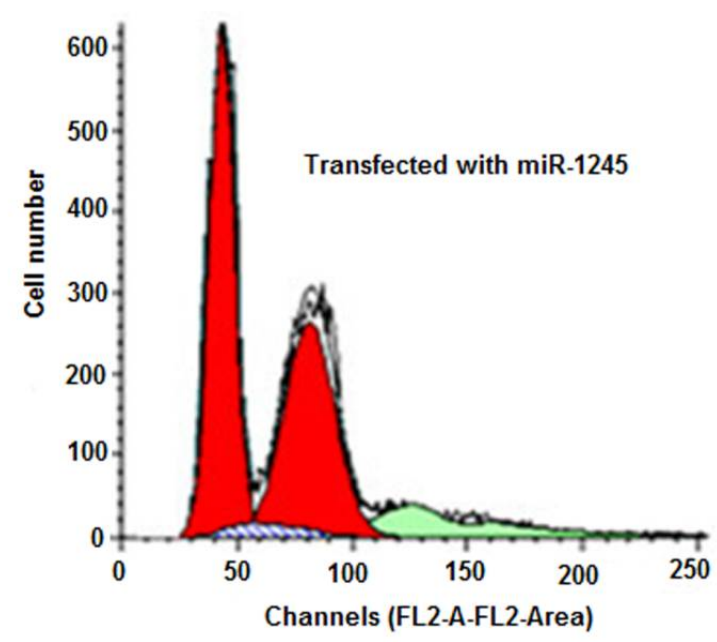

Figure 4: MiR-1254 overexpression triggers G2/M cell cycle arrest as determined by flow cytometery $(n=3)$ 

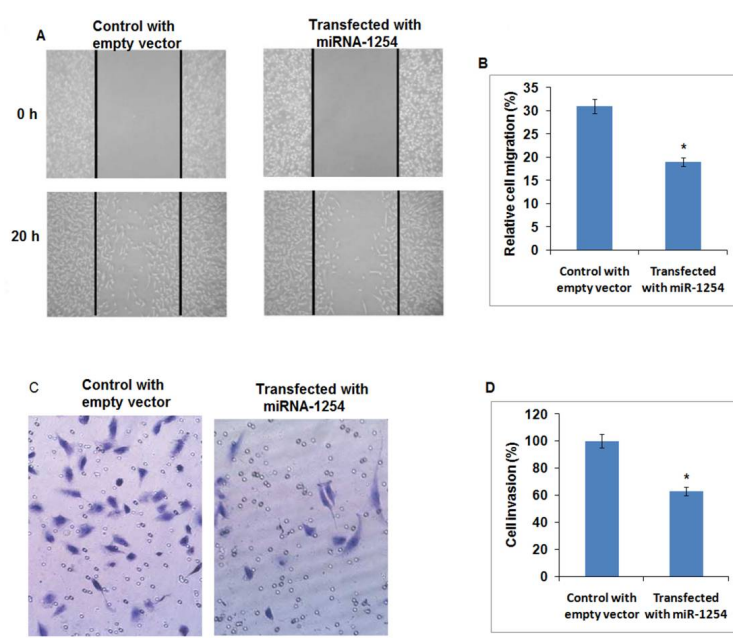

Figure 5: MiR-1254 overexpression inhibits $(A)$ cell migration (B) cell invasion as observed in wound healing and boyden chamber assays respectively. The experiments were carried out thrice in triplicates and expressed as mean $\pm \mathrm{SD}\left({ }^{*} p<0.05\right)$

\section{DISCUSSION}

MicroRNAs are small RNA molecules that do not code for any protein. They are involved in diverse cellular functions, and control some important processes such as cell cycle progression and regulation of gene expression [8]. These microRNAs have gained tremendous importance as therapeutic agents for the treatment of cancer [9]. They have been reported to show unique expression in wide array of cancers. The microRNAs have been reported to control cell proliferation, apoptosis, cell cycle arrest, metastasis, angiogenesis, migration and invasion of several types of cancer cells [10]. Hence, they are considered as important therapeutic targets for the treatment of cancers. Against this backdrop, the current study was designed to investigate the expression of miR-1254 in astrocytoma cell lines, and the role of miR-1254 in these cells.

It was observed that expression of miR-1254 was highly down-regulated in all the astrocytoma cell lines studied. The results are well supported by previous studies wherein miR-1254 was reported to be down-regulated in several types of cancers, including lung cancer [11,12]. In order to investigate the function of miR-1254 in astrocytoma, miR-1254 was transfected into SW1088 astrocytoma cells. The results showed that ectopic expression of miR-1254 induced apoptosis in SW 1088 astrocytoma cells, as was evident from the DAPI, EB/AO and annexin V/PI staining. Previously, miR-1254 was also reported to inhibit the proliferation of cancer cells which include but are not limited to colorectal cancer $[11,12]$.
MicroRNAs have also been reported to play a key role in progression of the cancer cells through different phases of the cell cycle [13]. The results of the present study showed that ectopic expression of miR-1254 caused G2/M cell arrest in the SW 1088 astrocytoma cells. Earlier investigations carried out on miR-1254 have revealed that it inhibited cell migration and invasion of cancer cells [14]. For instance, miR1254 has been reported to inhibit the migration and invasion of colorectal cancer by inhibiting PSMD10 [15]. Therefore, the effect of miR-1254 on the migration and invasion of SW 1008 astrocytoma cells was also investigated in the current study. The results showed that miR-1254 significantly inhibited migration and invasion of astrocytoma cells. The results strongly indicate that miR-1254 may be a therapeutic target for the treatment astrocytoma. This prospect merits further investigations.

\section{CONCLUSION}

The findings of this study show that miR-1254 is highly down-regulated in astrocytoma cell lines. Ectopic expression of miR-1254 causes inhibition of cell proliferation via induction of apoptosis and cell cycle arrest while miR-1254 inhibits migration and invasion of the astrocytoma cell lines. Therefore, miR-1254 is a potential therapeutic target for astrocytoma treatment, and deserves further investigations.

\section{DECLARATIONS}

\section{Conflict of interest}

No conflict of interest is associated with this work.

\section{Contribution of authors}

We declare that this work was done by the authors named in this article and all liabilities pertaining to claims relating to the content of this article will be borne by the authors. This study is designed and under supervised by Yan Wang. Gang Li prepared this manuscript and did most experiments with Dong-Gang Zhao. Lai Jiang, Yu-Hua Guan, Hua Tang, Gang Zheng, Xin Huang gave assistance in interpreting the data.

\section{REFERENCES}

1. Lu J, Getz G, Miska EA, Alvarez-Saavedra E. MicroRNA expression profiles classify human cancers. Nature 2005; 435(7043): 834.

2. Lewis $B P$, Burge $C B$, Bartel DP. Conserved seed pairing, often flanked by adenosines, indicates that thousands of 
human genes are microRNA targets. Cell 2005; 4: 120(1): 15-20.

3. Iorio MV, Ferracin M, Liu CG, Veronese A, Spizzo R, Sabbioni S, Magri E, Pedriali M, Fabbri M, Campiglio M, Ménard S. MicroRNA gene expression deregulation in human breast cancer. Cancer Res 2005; 65(16):70657070.

4. He L, Thomson JM, Hemann MT, Hernando-Monge E, Mu D, Goodson S, Powers S, Cordon-Cardo C, Lowe SW, Hannon GJ, Hammond SM. A microRNA polycistron as a potential human oncogene. Nature 2005; 435(7043): 828.

5. Jansson MD, Lund AH. MicroRNA and cancer. Molecular oncol 2012; 6(6): 590-610.

6. Zhu W, He J, Chen D, Zhang B, Xu L, Ma H, Liu X, Zhang $Y$, Le $H$. Expression of miR-29c, miR-93, and miR-429 as potential biomarkers for detection of early stage non-small lung cancer. PloS One 2014; 9(2): e87780.

7. Singh SK, Clarke ID, Terasaki M, Bonn VE, Hawkins C, Squire J, Dirks $P B$. Identification of a cancer stem cell in human brain tumors. Cancer Res 2003; 63(18): 5821 5828.

8. Iorio MV, Croce CM. MicroRNA dysregulation in cancer: diagnostics, monitoring and therapeutics. A comprehensive review. EMBO Molecular Med 2012; 4(3): 143-159.
9. Catto JW, Alcaraz A, Bjartell AS, White RD, Evans CP, Fussel S, Hamdy FC, Kallioniemi O, Mengual L, Schlomm $T$, et al. MicroRNA in prostate, bladder, and kidney cancer: a systematic review. Eur Urol 2011; 59(5): 671-681.

10. Xu C, Liu S, Fu H, Li S, Tie Y, Zhu J, Xing R, Jin Y, Sun $Z$, Zheng $X$. MicroRNA-193b regulates proliferation, migration and invasion in human hepatocellular carcinoma cells. Eur J Cancer 2010; 46(15): 2828-36.

11. Li $H$, Yang $T$, Shang $D$, Sun Z. miR-1254 promotes lung cancer cell proliferation by targeting SFRP1. Biomed Pharmacother 2017; 92: 913-918.

12. Li $Q$, Shen W, Li X, Zhang L, Jin X. The IncRNA n340790 accelerates carcinogenesis of thyroid cancer by regulating miR-1254. Amer J Translational Res 2017; 9(5): 2181.

13. Chen F, Hu SJ. Effect of microRNA-34a in cell cycle, differentiation, and apoptosis: A review. $J$ Biochemical Molecular Toxicology 2012; 26(2): 79-86.

14. Pu M, Li C, Qi X, Chen J, Wang Y, Gao L, Miao L, Ren J. MiR-1254 suppresses HO-1 expression through seed region-dependent silencing and non-seed interaction with TFAP2A transcript to attenuate NSCLC growth. PLoS Genetics 2017; 13(7): e1006896.

15. Chu YM, Peng HX, Xu Y, Yang DM, Zhou FL, Li J, Kuai $R$, Lin Y. MicroRNA-1254 inhibits the migration of colon adenocarcinoma cells by targeting PSMD10. J Digestive Dis 2017; 18(3): 169-178. 